\title{
Series: Cardiovascular outcome trials for diabetes drugs Empagliflozin and EMPA-REG OUTCOME
}

\author{
MILES FISHER
}

\begin{abstract}
EMPA-REG OUTCOME was an FDA-mandated cardiovascular outcome trial with empagliflozin and was the first completed trial with a sodium-glucose co-transporter-2 (SGLT2) inhibitor. EMPA-REG OUTCOME compared empagliflozin and placebo in 7,020 subjects with type 2 diabetes and established atherosclerotic cardiovascular disease. The results were astounding as EMPA-REG OUTCOME demonstrated superiority for major cardiovascular events (cardiovascular death, myocardial infarction, stroke) and cardiovascular deaths were significantly reduced, as was all-cause mortality. Hospitalisation for heart failure, which was a secondary outcome, was also significantly reduced. Later trials with SGLT2 inhibitors have demonstrated reductions in major adverse cardiovascular events (MACE) and hospitalisation for heart failure, and trials with glucagon-like peptide 1 receptor agonists have demonstrated reductions in MACE. Collectively, these trials could transform the management of people with type 2 diabetes.

Br J Diabetes 2020;20:138-141
\end{abstract}

Key words: diabetes, cardiovascular outcome trial, empagliflozin

\section{Introduction}

Licensing requirements for new anti-diabetes drugs changed in the USA and Europe in 2008 and 2012, and a dedicated randomised controlled cardiovascular outcome trial (CVOT) was usually required either before or after licensing.,2 This series describes and summarises the results of each of these CVOTs in the order in which they were published, describing the primary endpoint and important secondary outcomes from the principal publication, and directs attention to important subsequent publications of data from subgroups and post hoc analyses. EMPA-REG OUTCOME was the first published FDA-mandated cardiovascular outcome trial using a sodium-glucose co-transporter-2 (SGLT2) inhibitor. ${ }^{3}$ It came after three trials with dipeptidyl peptidase 4 (DPP-4) inhibitors ${ }^{4-6}$ and one

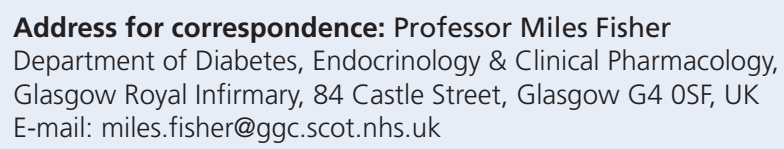

trial with the glucagon-like peptide 1 (GLP-1) receptor agonist lixisenatide had demonstrated safety but not cardiovascular benefit, ${ }^{7}$ and at a time when some commentators were raising questions as to whether the large cost of these trials was justified..$^{8,9}$

\section{Background}

Empagliflozin was approved in 2014 by the Food and Drug Administration (FDA) for use in the USA and by the European Medicines Agency (EMA) for use in Europe. Interim data from EMPA-REG OUTCOME were included in the new drug application to the FDA. The FDA initially rejected the new drug application for empagliflozin based on deficiencies at its main manufacturing facility. Empagliflozin was the third SGLT2 inhibitor to be approved by the FDA and EMA after dapagliflozin and canagliflozin, but was the first to complete a CVOT. At the time of publication of EMPA-REG OUTCOME in 2015 there were no published data on the cardiovascular safety of empagliflozin. In 2016 a meta-analysis of prospectively adjudicated cardiovascular outcomes from eight trials of empagliflozin, including EMPA-REG OUTCOME, demonstrated a significant reduction in major adverse cardiovascular events (MACE) plus (cardiovascular death, non-fatal myocardial infarction, nonfatal stroke, hospitalisation for unstable angina). ${ }^{10}$ The results were still significant when the analysis was performed excluding EMPAREG OUTCOME.

\section{EMPA-REG OUTCOME}

A paper describing the rationale, design and baseline characteristics of EMPA-REG OUTCOME was published in $2014 .{ }^{11}$ The principal EMPA-REG OUTCOME results were presented in 2015 at the meeting of the European Association for the Study of Diabetes (EASD), where it received a standing ovation, and published simultaneously in the New England Journal of Medicine. ${ }^{3}$ The design of the study and key baseline characteristics are described in Box 1. Two doses of empagliflozin were included (10 $\mathrm{mg}$ and $25 \mathrm{mg}$ ) and the results were pooled for analysis.

In EMPA-REG OUTCOME there was a significant reduction in MACE with empagliflozin, demonstrating superiority versus placebo (Figure 1, Box 2). In the pooled empagliflozin group there were statistically significant reductions in cardiovascular death, hospitalisation for heart failure and death from any cause. There were no significant differences in the rates of myocardial infarction or stroke, although a non-significant increase in strokes was observed. The rate of genital infection was increased with empagliflozin and there was no increase in other adverse events including urinary tract 
Box 1 Key features of EMPA-REG OUTCOME 3,11

- EMPA-REG OUTCOME compared empagliflozin $10 \mathrm{mg}$ and $25 \mathrm{mg}$ versus placebo for a median observation time of 3.1 years in 7,020 subjects

- Mean age of subjects was 63 years and $57 \%$ of subjects had a duration of diabetes over 10 years

- Mean baseline $\mathrm{HbA}_{1 \mathrm{c}}$ was $8.1 \%(65 \mathrm{mmol} / \mathrm{mol})$

- $99 \%$ of subjects had established atherosclerotic disease, $47 \%$ prior myocardial infarction, 25\% prior coronary artery bypass grafting, $24 \%$ prior stroke and $10 \%$ investigator-reported heart failure, but this diagnosis was not well characterised

- $74 \%$ of subjects were on metformin, $42 \%$ sulfonylureas, $4 \%$ thiazolidinediones, $48 \%$ insulin

Figure 1. 3.1-year event rates (in \%) comparing empagliflozin and placebo for major adverse cardiovascular events (MACE), total mortality, cardiovascular mortality, non-fatal myocardial infarction, non-fatal stroke, and hospitalisation for heart failure

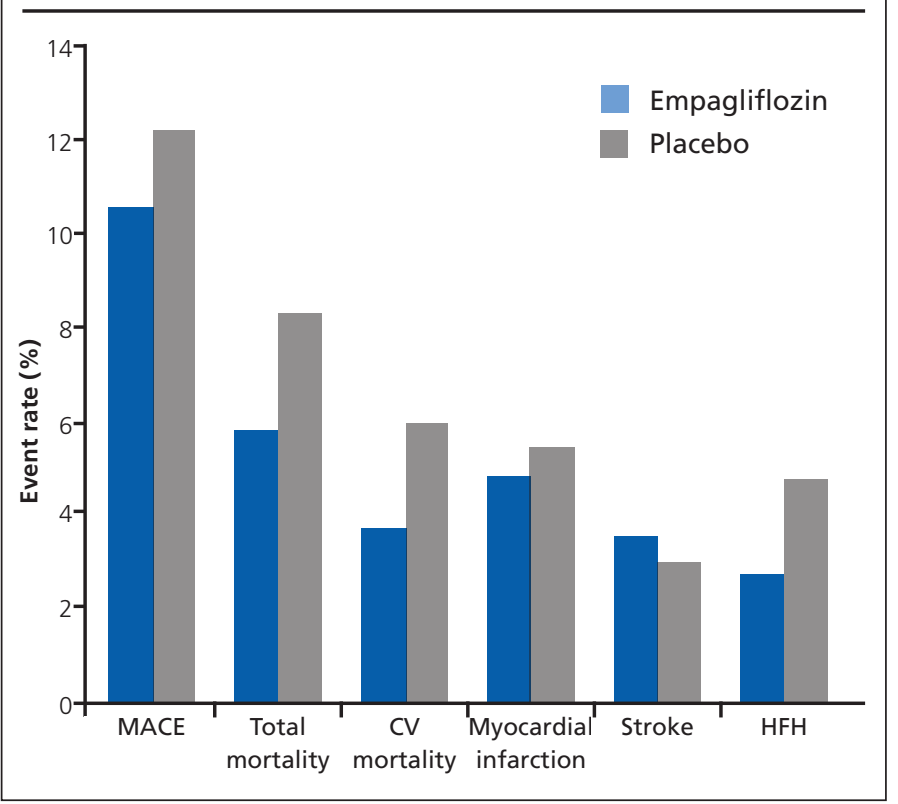

infection. There were four episodes of diabetic ketoacidosis in the pooled empagliflozin group and one in the placebo group.

\section{Other results from EMPA-REG OUTCOME}

Further publications from EMPA-REG OUTCOME are detailed in Box 2. 10\% of subjects in EMPA-REG OUTCOME had investigatorreported heart failure at baseline. ${ }^{3}$ Further analysis of heart failure data demonstrated significant reductions in hospitalisation for heart failure in patients with and without baseline heart failure, and across categories of medications to treat diabetes or heart failure. ${ }^{12}$ Death from heart failure was also significantly reduced with empagliflozin.

Further analysis of renal outcomes showed a reduction in incident or worsening nephropathy, a reduction in doubling of serum creatinine, and a reduction in renal replacement therapy with empagliflozin. ${ }^{13}$
Box 2 Results of the EMPA-REG OUTCOME trial

Principal result

- Significant reduction in MACE, cardiovascular death, hospitalisation for heart failure and death from any cause ${ }^{3}$

Other results from EMPA-REG OUTCOME

- Hospitalisation for heart failure and other heart failure outcomes were reduced with empagliflozin in patients with and without investigator-reported heart failure at baseline $e^{3,12}$

- Use of empagliflozin was associated with slower progression of kidney disease and lower rates of clinically relevant renal events than placebo ${ }^{3,13}$

- In EMPA-REG OUTCOME there was a non-significant increase in strokes. ${ }^{3}$ Further analysis showed this numerical difference was primarily because of 18 patients with a stroke event more than 90 days after the last dose of empagliflozin versus 3 patients in the placebo group ${ }^{19}$

- In an exploratory mediation analysis, changes in markers of plasma volume were the most important mediators of the reduction of cardiovascular death with empagliflozin 20

- In patients with peripheral arterial disease, empagliflozin reduced cardiovascular death and all-cause mortality, with no increase in the risk of lower limb amputation ${ }^{21}$

- Using actuarial methods and assuming that the benefits of empagliflozin remain consistent with long-term use, it was estimated that empagliflozin improved survival by $1-5$ years $^{22}$

- In a post hoc exploratory analysis, hypoglycaemia was associated with an increased risk of heart failure and myocardial infarction. Hypoglycaemia risk was not increased with empagliflozin and incident hypoglycaemia did not attenuate its cardio-protective effects $^{23}$

- Improvements in liver function tests with empagliflozin were potentially consistent with reductions in liver fat ${ }^{24}$

\section{Discussion}

Based on the pleiotropic effects of SGLT2 inhibitors on cardiovascular risk markers, including reductions in $\mathrm{HbA}_{1 \mathrm{c}}$, body weight and blood pressure, the EMPA-REG OUTCOME investigators hypothesised that empagliflozin might reduce cardiovascular risk in patients with type 2 diabetes. ${ }^{11}$ The strongly positive results of EMPA-REG OUTCOME, however, were not predicted and the significant reductions in cardiovascular deaths and hospitalisation for heart failure were particularly unexpected. The reduction in the risk of hospitalisation for heart failure and of cardiovascular death was observed early in EMPA-REG OUTCOME, and since the publication of EMPA-REG OUTCOME there have been many reviews speculating on the possible mechanisms of benefit, as these were not studied in EMPA-REG OUTCOME. Similar reductions in hospitalisation for heart failure were subsequently observed in CANVAS and DECLARE-TIMI 58. ${ }^{14,15}$ Meta-analysis of EMPA-REG OUTCOME, CANVAS and DECLARETIMI 58 has confirmed reductions in heart failure, cardiovascular deaths and renal composites. ${ }^{16}$ Significant reductions in MACE have been seen in patients with existing atherosclerotic cardiovascular disease, as was included in EMPA-REG OUTCOME, but MACE benefit does not appear to occur in patients without atherosclerotic disease. ${ }^{16}$ Following analysis of EMPA-REG OUTCOME, the DAPA-HF trial was initiated in patients with severe heart failure and well-characterised baseline ejection fractions, including a majority of subjects who did not have diabetes. This 


\section{Key messages}

- EMPA-REG OUTCOME was the fifth published cardiovascular outcome trial of a diabetes drug, the first using a SGLT2 inhibitor, comparing empagliflozin and placebo

- In EMPA-REG OUTCOME, empagliflozin significantly reduced major adverse cardiovascular events, cardiovascular death and hospitalisation for heart failure

- Subsequent trials with canagliflozin and dapagliflozin demonstrated significant reductions in heart failure events

- The possible mechanisms of benefit of empagliflozin and other SGLT2 inhibitors are currently under investigation

confirmed significant reductions in hospitalisation for heart failure, cardiovascular deaths and total mortality in this different patient group. ${ }^{17}$

The FDA has recently withdrawn the guidance from $2008^{1}$ and are consulting on new draft guidance for evaluating the safety of new drugs for improving glycaemic control. ${ }^{18}$ The FDA comment that none of the CVOTs has identified an increased risk of ischaemic events, and that some have instead identified a reduced risk for cardiovascular events. The draft recommendations for new drugs include a safety database of at least 4,000 patient-years of exposure to the new drug in phase 3 clinical trials, 500 patients with chronic kidney disease, 600 patients with established cardiovascular disease and 600 patients aged $>65$ years. They recommend that sponsors should use rigorous methods for the collection of adverse cardiovascular events and assess them for adjudication. The need for a dedicated cardiovascular safety trial is removed from the draft, which would mean that, in the future, landmark trials like EMPAREG OUTCOME may be performed at the discretion of the sponsors and clinical investigators but would not be obligatory.

Conflict of interest The author has received personal fees from AstraZeneca, Boehringer Ingelheim, Eli Lilly, Lexicon, MSD, NAPP, Novo Nordisk and Sanofi, outside the submitted work.

\section{Funding None.}

\section{References}

1. Food and Drug Administration. Guidance for industry. Diabetes mellitus - evaluating cardiovascular risk in new antidiabetic therapies to treat type 2 diabetes. Silver Spring, MD: US Department of Health and Human Services, Food and Drug Administration, Center for Drug Evaluation and Research (CDER), 2008.

2. European Medicines Agency (EMA). Guideline on clinical investigation of medicinal products in the treatment or prevention of diabetes mellitus. London: EMA, 2012. Available from: http://www.ema.europa.eu/docs/ en_GB/document_library/Scientific_guideline/2012/06/ WC500129256.pdf (accessed 1 March 2020).

3. Zinman B, Wanner C, Lachin JM, et al, for the EMPA-REG OUTCOME investigators. Empagliflozin, cardiovascular outcomes, and mortality in type 2 diabetes. N Engl J Med 2015;373:2117-28. https://doi.org/

10.1056/NEJMoa1504720

4. Scirica BM, Bhatt DL, Braunwald E, et al, for the SAVOR-TIMI 53 Steering Committee and Investigators. Saxagliptin and cardiovascular outcomes in patients with type 2 diabetes mellitus. N Engl J Med 2013;369:131726. https://doi.org/10.1056/NEJMoa1307684

5. White WB, Cannon CP, Heller SR, et al, for the EXAMINE Investigators. Alogliptin after acute coronary syndrome in patients with type 2 diabetes. N Engl J Med 2013;369:1327-35. https://doi.org/10.1056/NEJMoa1305889

6. Green JB, Bethel A, Armstrong PW, et al, for the TECOS Study Group. Effect of sitagliptin on cardiovascular outcomes in type 2 diabetes. N Engl J Med 2015;373:232-42. https://doi.org/10.1056/NEJMoa1501352

7. Pfeffer MA, Claggett B, Diaz R, et al, for the ELIXA Investigators. Lixisenatide in patients with type 2 diabetes and acute coronary syndrome. N Engl J Med 2015;373:2247-57. https://doi.org/10.1056/NEJMoa1509225

8. Hirshberg B, Katz A. Insights from cardiovascular outcome trials with novel antidiabetes agents: what have we learned? An industry perspective. Curr Diab Rep 2015;15:87. https://doi.org/10.1007/s11892-015-0663-9

9. Steg PG, Roussel R. Randomized trials to evaluate cardiovascular safety of antihyperglycemic medications. A worthwhile effort? Circulation 2016;134:571-3. https://doi.org/10.1161/CIRCULATIONAHA.116.021914

10. Salsali A, Kim G, Woerle HJ, et al. Cardiovascular safety of empagliflozin in patients with type 2 diabetes: a meta-analysis of data from randomized placebo-controlled trials. Diabetes Obes Metab 2016;18:1034-40. https://doi.org/10.1111/dom.12734

11. Zinman B, Inzucchi SE, Lachin JM, et al. Rationale, design, and baseline characteristics of a randomized, placebo-controlled cardiovascular outcome trial of empagliflozin (EMPA-REG OUTCMETM). Cardiovasc Diabeto/ 2014;13:102. https://doi.org/10.1186/1475-2840-13-102

12. Fitchett $D$, Zinman $B$, Wanner $C$, et al, on behalf of the EMPA-REG OUTCOME Trial Investigators. Heart failure outcomes with empagliflozin in patients with type 2 diabetes at high cardiovascular risk: results of the EMPA-REG OUTCOME TRIAL@. Eur Heart J 2016;37:1526-34. https://doi.org/10.1093/eurheartj/ehv728

13. Wanner C, Inzucchi SE, Lachin JM, et al, for the EMPA-REG OUTCOME Investigators. Empagliflozin and progression of kidney disease in type 2 diabetes. N Engl J Med 2016;375:323-34. https://doi.org/10.1056/NEJMoa1515920

14. Neal B, Perkovic V, Mahaffey KW, et al, for the CANVAS Program Collaborative Group. Canagliflozin and cardiovascular and renal events in type 2 diabetes. N Engl J Med 2017;377:644-57. https://doi.org/ 10.1056/NEJMoa1611925

15. Wiviott SD, Bonaca MP, Mosenzon O, et al, for the DECLARE-TIMI 58 Investigators. Dapagliflozin and cardiovascular outcomes in type 2 diabetes. N Engl J Med 2019;380:347-57. https://doi.org/10.1056/NEJMoa1812389

16. Zelniker TA, Wiviott SD, Raz I, et al. SGLT2 inhibitors for primary and secondary prevention of cardiovascular and renal outcomes in type 2 diabetes: a systematic review and meta-analysis of cardiovascular outcome trials. Lancet 2019;393:31-9. https://doi.org/10.1016/S01406736(18)32590-X

17. McMurray JJV, Solomon SD, Inzucchi SE, et al, for the DAPA-HF Trial Committees and Investigators. Dapagliflozin in patients with heart failure and reduced ejection fraction. N Engl J Med 2019;381:1995-2008. https://doi.org/10.1056/NEJMoa1911303

18. Food and Drug Administration. Type 2 diabetes mellitus: evaluating the safety of new drugs for improving glycemic control. Guidance for industry. Silver Spring, MD: US Department of Health and Human Services, Food and Drug Administration, Center for Drug Evaluation and Research (CDER), 2020. Available from https://www.federalregister.gov/documents/2020/03/10/2020-04877/type-2-diabetes-mellitus-evaluatingthe-safety-of-new-drugs-for-improving-glycemic-control-draft (accessed 10 June 2020).

19. Zinman B, Inzucchi SE, Lachin JM, et al, on behalf of the EMPA-REG OUTCOME Investigators (Empagliflozin Cardiovascular Outcome Event Trial in Type 2 Diabetes Mellitus Patients). Empagliflozin and cerebrovascular events in patients with type 2 diabetes mellitus at high cardiovascular risk. Stroke 2017;48:1218-25. https://doi.org/10.1161/ STROKEAHA.116.015756

20. Inzucchi SE, Zinman B, Fitchett D, et al. How does empagliflozin reduce 
cardiovascular mortality? Insights from a mediation analysis of the EMPA-REG OUTCOME Trial. Diabetes Care 2018;41:356-63. https://doi.org/10.2337/dc17-1096

21. Verma S, Mazer CD, Al-Omran M, et al. Cardiovascular outcomes and safety of empagliflozin in patients with type 2 diabetes mellitus and peripheral artery disease: a subanalysis of EMPA-REG OUTCOME. Circulation 2017;136:405-7. https://doi.org/10.1161/CIRCULATIONAHA.117.032031

22. Claggett $B$, Lachin JM, Hantel S, et al. Long-term benefit of empagliflozin on life expectancy in patients with type 2 diabetes mellitus and established cardiovascular disease. Survival estimates from the EMPA-REG OUTCOME Trial. Circulation 2018;138:1599-1601.
https://doi.org/10.1161/CIRCULATIONAHA.118.033810

23. Fitchett $D$, Inzucchi SE, Wanner $C$, et al. Relationship between hypoglycaemia, cardiovascular outcomes, and empagliflozin treatment in the EMPA-REG OUTCOME $®$ trial. Eur Heart J 2020;41:209-17. https://doi.org/10.1093/eurheartj/ehz621

24. Sattar N, Fitchett D, Hantel S, George JT, Zinman B. Empagliflozin is associated with improvements in liver enzymes potentially consistent with reductions in liver fat: results from randomised trials including the EMPA-REG OUTCOME $®$ trial. Diabetologia 2018;61:2155-63. https://doi.org/10.1007/s00125-018-4702-3

\section{YDEF NEWS}

This is only a brief update - because COVID has stopped the majority of our courses and we are trying to adapt to the virtual environment we find ourselves in. Despite this, the YDEF committee is continuing to develop courses and opportunities for SpRs and young consultants. Little has changed since the previous update except perhaps a few extra grey hairs from the last few months!

Firstly, we were pleased to be able to support the speciality advisory committee at the Royal College of Physicians in the development of the new curriculum for trainees. The draft version should be available for sharing in the coming weeks. We also have taken this opportunity to survey trainees nationally to try providing evidence of problems we encounter with training, or those that might develop in the future if access to tertiary centres is reduced in the shift to Internal Medicine Training (the new Core Medical Training) and the introduction of IMT3. We have some provisional results which we are hoping to publish and share in the coming months and that we hope provokes thought about what needs to be done in every area of the country to provide some protected training without the oftentime-consuming commitment to general medicine rotas or ward work.

We were really pleased to be able to run an Obesity webinar jointly with the Association for the Study of Obesity (ASO) - this was well attended and greatly received. This is an important topic and something which are planning to focus more resources on in future. Our initial plan for a 2-ay course was scuppered by the virus, but we plan to go ahead with this in some form (virtual or in-person) in 2021.

COVID has also allowed us to work more closely with $A B C D$. We are really excited about our joint venture, supported by Lilly pharmaceuticals, for monthly webinars aimed at Diabetes \& Endocrine SpRs. We hope this will fill the gaps left from the training opportunities that we have lost due to COVID rotas and general medicine on-call commitments.

Finally, another joint venture. We are really pleased that alongside the British Journal of Diabetes we are launching a Quality Improvement Project award. We have all had to make significant changes to our services and some of the results have been impressive. There is no better way of sharing your work than writing these up and submitting for consideration (and there is an associated prize of $\mathrm{f} 250$ for the top 3 pieces or work as well as publication!). This is a fantastic opportunity for an SpR, early consultant or diabetes MDT member and we really hope people submit their projects and inspire other centres to think about how they might improve their services as well!

Dr Tim Robbins on behalf of YDEF Committee University Hospitals Coventry \& Warwickshire NHS Trust, UK Contact:drtrobbins@gmail.com

\section{YDEF is dedicated to all diabetes and endocrine trainees and is open for new members to register on our website. Take advantage of our regular newsletters and up-to-date advertising of a wide variety of courses and meetings to complement your training.}

\section{As always, we are continuously looking to develop and propagate our specialty so do not hesitate to contact us if you have any suggestions or questions!}

